MATEC Web of Conferences 6, 03004 (2013)

DOI: $10.1051 /$ matecconf/20130603004

(C) Owned by the authors, published by EDP Sciences, 2013

\title{
Neutron radiography of heated high-performance mortar
}

\author{
B. Weber ${ }^{1}$, M. Wyrzykowski ${ }^{1}$, M. Griffa ${ }^{1}$, S. Carl ${ }^{1}$, E. Lehmann ${ }^{2}$ and P. Lura ${ }^{1,3}$ \\ ${ }^{1}$ Empa, Swiss Federal Laboratories for Materials Science and Technology, \\ 8600 Dübendorf, Switzerland \\ 2 Paul Scherrer Institut, 5232 Villigen PSI, Switzerland \\ 3 Institute for Building Materials (IfB), ETH, 8093 Zurich, Switzerland
}

\begin{abstract}
Neutron radiography was applied to investigate the water distribution in mortar samples heated from one side to $600{ }^{\circ} \mathrm{C}$. In mortar, aggregates and anhydrous cement are almost transparent to neutrons, while hydration products and water-filled capillary pores bear the largest attenuation. The evolution of the moisture profile shows a sharp dehydration front and accumulation of water due to condensation of water vapor behind this front.
\end{abstract}

\section{INTRODUCTION}

High-Performance Concrete is becoming widespread, especially for prefabricated concrete elements. However, under fire exposure, these elements are prone to explosive spalling. Explosive spalling of the concrete cover exposes the reinforcement and may lead to premature failure of concrete structures. One of the mechanisms leading to spalling is the pressure buildup of water vapor during heating. Vapor is produced by evaporation of initial moisture in the pores as well as by dehydration of different phases present in the cementitious matrix. Depending on the permeability, vapor may escape or it may increase the gas pressure in the pores. In order to better understand this complex mechanism, it is paramount to be able to follow the moisture distribution in concrete during exposure to fire. For this purpose, moisture profiles in the concrete need to be followed quantitatively and non-destructively in real time. Recently, a dedicated NMR setup was developed for this purpose [1].

Another effective method to measure water distribution in concrete is neutron radiography. Measurements of water content and 2D spatial distribution by radiography are based upon the transmission of a radiation beam through the sample. Pixel-wise changes in beam intensity recorded on a plane detector parallel to the sample allow for estimating the local changes in water content.

The contrast in a radiograph is given by the different effective attenuation cross-sections of the distinct chemical elements for the type of radiation exploited. On a microscopic (atomic) level, the attenuation is determined by the effective quantum mechanics cross-section $\sigma$ (expressed in barns, 1 barn $=10^{-28} \mathrm{~m}^{2}$ ) for the different types of interactions between the chemical elements and the radiation beam (absorption, coherent and incoherent scattering). For X-rays, the effective attenuation cross-section of an element is roughly proportional to its atomic number. In the case of neutrons, it is not simply proportional to the atomic number but depends upon the nuclear structure. On a macroscopic level, the effective attenuation cross-section $\Sigma$ is the product of the atom density of chemical elements contained in the specimen (in atoms $/ \mathrm{cm}^{3}$ ) and their microscopic cross-sections. The macroscopic effective attenuation cross-section has thus the physical dimension of $1 / \mathrm{cm}$. Since hydrogen has a large neutron scattering cross-section (about 30 barn for thermal neutrons, at $25 \mathrm{meV}$ of energy), a neutron

This is an Open Access article distributed under the terms of the Creative Commons Attribution License 2.0, which permits unrestricted use, distribution, and reproduction in any medium, provided the original work is properly cited. 


\section{MATEC Web of Conferences}
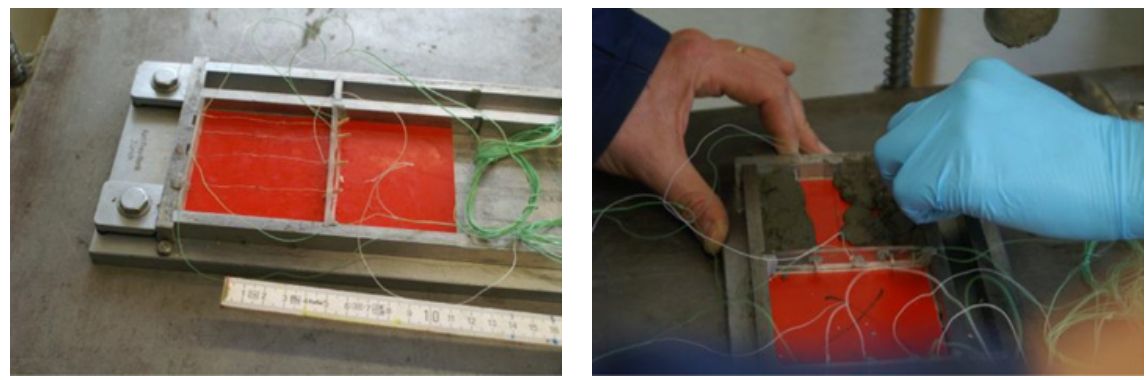

Figure 1. Casting of mortar samples.

beam is highly attenuated by substances containing hydrogen. This feature makes neutron radiography a very sensitive tool for following changes in water content of a material.

In concrete, aggregates and anhydrous cement are almost transparent to neutrons, while hydration products and water-filled capillary pores bear the largest attenuation. Neutron radiography and tomography, due to high sensitivity for water, was applied by several research groups to study moisture transport in cementitious materials (e.g. [2-8]). The application of neutron radiography to study water transport in cementitious materials subjected to high temperatures was suggested already in [2]; however, this idea was not followed up until recently [9].

In this study, neutron radiography was applied to investigate the water distribution in mortar samples heated from one side to $600^{\circ} \mathrm{C}$. The experiments were performed at the NEUtron Transmission RAdiography (NEUTRA) beamline of the Paul Scherrer Institute (PSI) in Villigen, Switzerland. This beamline is fed by the Swiss Neutron Spallation Source (SINQ) and is operated with neutrons within a thermal spectrum characterized by a Maxwell-like probability density function, with a most probable energy level of about $25 \mathrm{meV}$ [10].

\section{MATERIALS AND METHODS}

The cross-section of the samples was chosen to fit into the field of view of the neutron beam, which is $150 \times 150 \mathrm{~mm}^{2}$. The thickness of the samples had to be chosen such that it did not produce too large attenuation, thus avoiding the need of long exposure time of the camera to achieve a sufficient signal-to-noise ratio. Generally, a smaller thickness requires a shorter exposure time and creates fewer artifacts due to scattering. Since this was the first experiment of this kind at this facility, a relatively small thickness was chosen, even though a thicker sample might have been more realistic. Considering these boundary conditions, three mortar samples of dimensions $80 \times 80 \times 10 \mathrm{~mm}^{3}$ were prepared.

The mortar was a high-performance mixture with a water-to-cement ratio of 0.30 including a superplasticizer and sand of $0.25-1.0 \mathrm{~mm}$ size. It was mixed using a vacuum mixer. Thermocouples of type $\mathrm{K}$ were fixed in the molds and the mortar was carefully filled into the mold while shaking it on a vibration table (Figure 1). After demolding at 1 day, the samples were sealed in plastic bags and stored at $20^{\circ} \mathrm{C}$ and $95 \%$ RH humidity for 4 weeks.

In order to obtain one-dimensional temperature and moisture fields, the specimens were insulated against moisture and heat loss, except from the bottom and top sides. The installation of the insulation layers is shown in Fig. 2. Aluminum foil was glued on the front and the back face to avoid vapor loss (aluminum is almost transparent to neutrons). The aluminum foil prevents the sample from drying out through the front and back face but it does not provide a tight sealing that allows for a large pressure build-up. The lateral faces were covered with glass foam, and glass foam sheets were also attached to the front and the back face with aluminum clamps. 

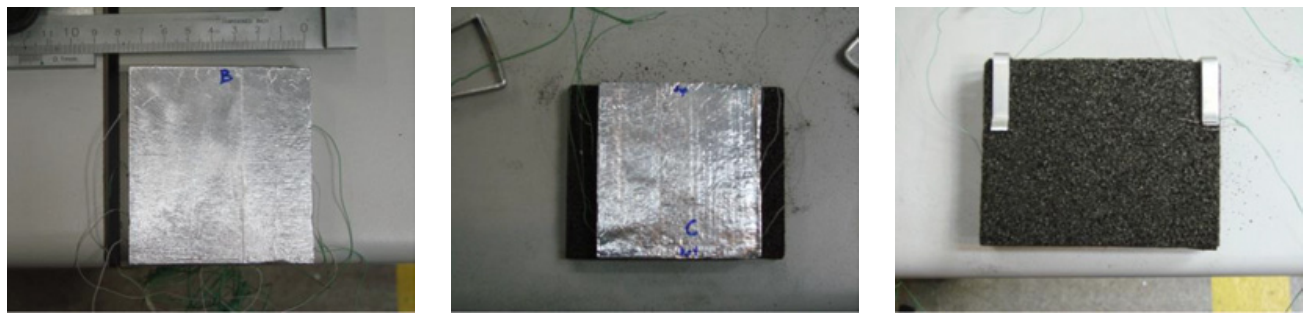

Figure 2. Insulation layers applied to mortar samples.
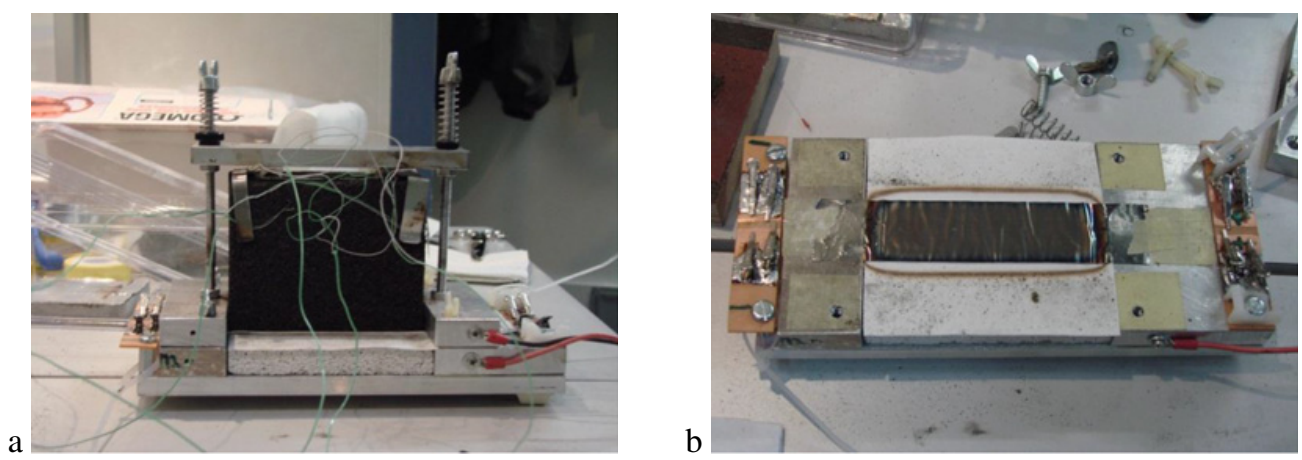

Figure 3. The test setup used to heat the concrete from the bottom side while in the neutron beam, b) lower part with heating device.

The test setup used to heat the concrete from the bottom side while in the neutron beam is shown in Fig. 3a). It consists basically of a frame for holding the sample in place by pressing a horizontal beam down by two springs. The samples were heated with a metal foil lying underneath the sample and exposed to a high electrical current ( $25 \mathrm{~A}, 12 \mathrm{~V}$ ) controlled by a pair of thermocouples (Figure $3 \mathrm{~b}$ ). The same heating device was also employed in [11] to study dehydration and moisture transport in gypsum plaster boards.

\section{IMAGE ANALYSIS}

The neutron intensity recorded by the camera can be described by the Beer-Lambert law:

$$
I(x, y)=I_{0}(x, y) \mathrm{e}^{-\int_{\text {thickness }} \Sigma(x, y, z) d z}
$$

where $I_{0}$ is the intensity of the incident neutron beam and $\Sigma$ is the effective attenuation cross-section, which depends on the sample material. The integral is evaluated through the thickness of the sample at each pixel in the $x-y$-image plane. In our experiment, most of the attenuation is due to water, and all the change in attenuation will be due to differences in water content in time. Water is present as capillary water, adsorbed water and chemically bound water. Water vapor gives almost no contrast due to its low density. If we assume that water in all these forms has the same attenuation coefficient, we can use a two-layer model, where one layer represents the water in all its different forms and the other layer represents all other materials, i.e. anhydrous cement and aggregates. Then Equation (1) can be rewritten as

$$
I(x, y)=I_{0}(x, y) \mathrm{e}^{-\Sigma_{w} d_{w}(x, y)^{-} \Sigma_{c} d_{c}(x, y)}
$$




\section{MATEC Web of Conferences}

where now $\Sigma_{w}$ and $\Sigma_{c}$ are the effective attenuation cross-sections for the "water layer" and for the "cementitious layer", respectively, and $d_{w}$ and $d_{c}$ are equivalent thicknesses of these layers. If we assume that only the "water layer" changes its thickness during the experiment, Equation (2) describes the observed intensity $I(x, y, t)$ in each pixel as a function of the local equivalent thickness of the "water layer" $d_{w}(x, y, t)$. By writing Equation (2) for the initial time $t_{0}$ and the general time $t$ we can find the change in equivalent water thickness by

$$
\Delta d_{w}(x, y, t)=-\frac{1}{\Sigma_{w}} \ln \frac{I(x, y, t)}{I\left(x, y, t_{0}\right)}
$$

where $\Delta d_{w}(x, y, t)=d_{w}(x, y, t)-d_{w}\left(x, y, t_{0}\right)$. The effective attenuation cross-section for water at the NEUTRA instrument using a ${ }^{6} \mathrm{Li}$ scintillator is $\Sigma_{w}=3.5 / \mathrm{cm} \mathrm{[12].}$

To account for artifacts during data acquisition, corrections are usually made on the observed intensity $I(x, y, t)$ before applying Equation (3). In this paper, we are mainly interested in a semiquantitative analysis. Only some simple corrections were thus applied:

- The dark current correction accounts for background noise of the CCD camera pixels, which can be determined by taking a few reference images without a neutron beam.

- The intensity correction accounts for the temporal variability of the incident neutron beam during the experiment. A scaling factor is applied to the recorded image after subtracting the dark current correction. The scaling factor is determined such that the mean intensity in a region outside the specimen (blue rectangle in Figure 4 b) remains constant over time.

- The black body correction accounts for the neutron scattering of the overall experimental environment. This effect can be determined by taking a few reference images while blocking the field of view from the neutron beam with a highly absorbing screen, typically made of boronated polyethylene. The black body correction is subtracted from the scaled image.

For a fully quantitative analysis, other effects would have to be corrected for. These effects are in particular the scattering of neutrons by the specimen, and the incident beam's energy spectrum effects. These effects have to be determined with a numerical simulation of the neutron transmission through the sample in an iterative way $[12,13]$.

\section{RESULTS}

In this paper we only show results of one test, the other results being in principle identical.

Before starting the heating process, two initial radiographs were taken, one with X-rays and one with neutrons. These two radiographs are shown in Figure 4. For both radiographs, the image size is $1385 \times 1023$ pixels, as indicated by the axes on the top and on the left of the images. The nominal pixel size is about $100 \mu \mathrm{m}$. A physical coordinate system in $\mathrm{mm}$ is also shown with axes at the bottom and on the left. The specimen is the blue square extending from about $-40 \mathrm{~mm}$ to $+40 \mathrm{~mm}$ on the $\mathrm{x}$-axis and from $0 \mathrm{~mm}$ to about $80 \mathrm{~mm}$ on the y-axis. The yellow bars to the left and the right of the sample are the glass foam with very low neutron absorption. The dark blue regions on both sides and at the bottom are boronated polyethylene blocks that surround the sample to reduce neutron scattering.

The X-ray radiograph shown on the left (Fig. 4a) makes metal parts visible, in particular the position of the thermo-elements (indicated by red squares). Also visible are the half-moon shaped aluminum pieces holding the sample from above and the clamps fixing the glass foam at the top on both sides outside the sample. The neutron radiograph on the right (Fig. 4b) shows the drying front after 30 minutes of heating (raw image). The region indicated by the blue rectangle near the left boundary is used to normalize the intensity of the neutron beam (intensity correction). The material in this region supposedly does not undergo any change during the experiment. The region indicated by the red rectangle in the center of the specimen is used for determining the water content profile. Since drying in this region is an almost one-dimensional process, the intensities at each height are averaged in the horizontal direction within this window. This averging improves the accuracy of the profile. 

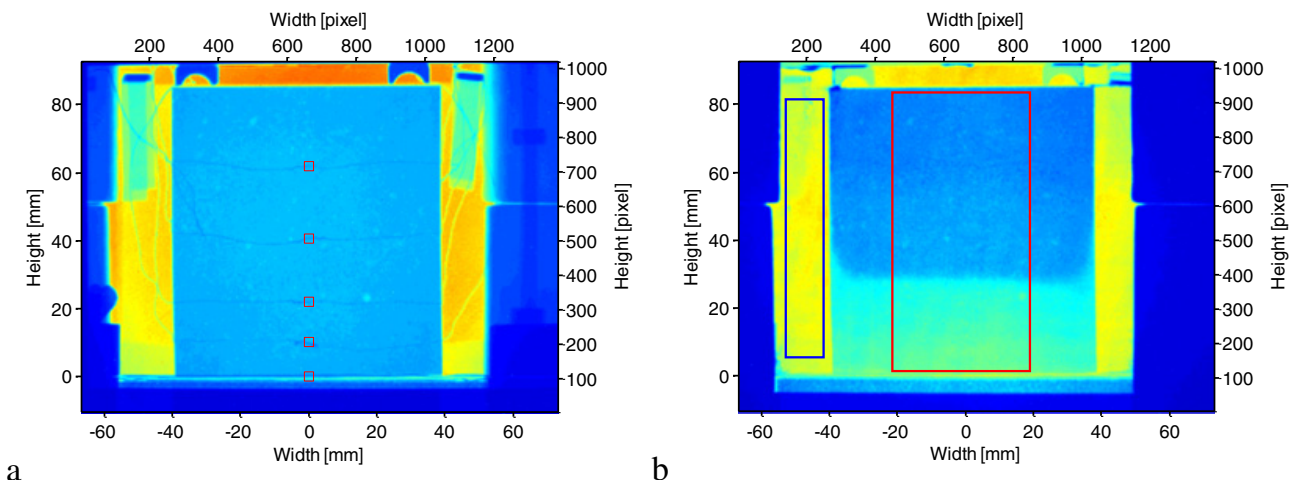

Figure 4. a) X-ray radiograph of the sample, showing the position of the thermocouples (small red squares). b) Neutron radiograph of the sample after 30 minutes of heating. Both radiographs are color-coded.
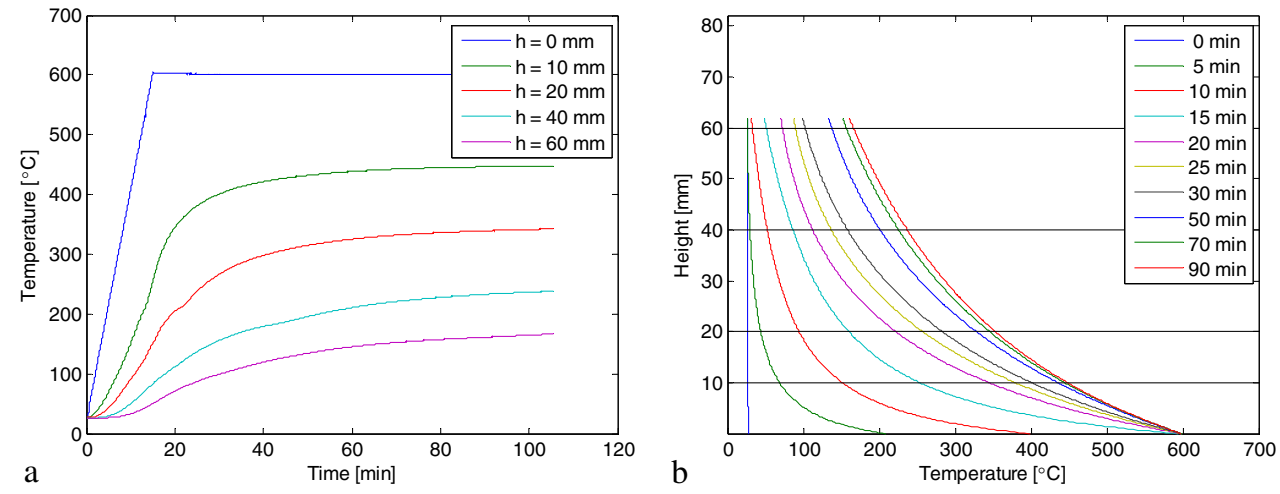

Figure 5. a) Temperature development as a function of time measured at the five thermocouples. b) Temperature profiles over the height of the specimen (obtained by interpolating the values measured at the five thermocouples).

Images were acquired every $17 \mathrm{~s}$ with an exposure time of $10 \mathrm{~s}$.

The temperatures measured at the five thermocouples are plotted in Figure 5a as a function of time. The bottom thermocouple is actually measuring the temperature of the heating foil and is used for controlling the heating temperature. A linear temperature rise up to $600^{\circ} \mathrm{C}$ in 15 minutes was specified there. Figure $5 \mathrm{~b}$ shows the temperature profiles over the height of the specimen obtained by interpolating the values measured at the five thermocouples. Note that the time increments are 5 minutes during the first 30 minutes and 20 minutes thereafter. Also note that with only five thermocouples (position indicated by dashed horizontal lines), the interpolated profiles can only give a global picture of the temperature field in the specimen, without too much detail. Especially near the heating foil, the resolution is not fine enough to capture the exact temperature front at the beginning of the heating process.

Figure 6a shows the profiles of the water loss during heating. The time increments are 5 minutes during the first 30 minutes and 10 minutes thereafter. The loss of water content observed in the neutron radiograph comprises the adsorbed, the chemically bound, and the free capillary water.

Near the heating device, drying of capillary water takes place quite quickly. The reversal in the first few millimeters near the bottom is probably due to the fact, that at the bottom surface the cement paste is already partially dried before the start of the experiment. Drying also occurred on the top of the sample, where no insulation was present. 


\section{MATEC Web of Conferences}
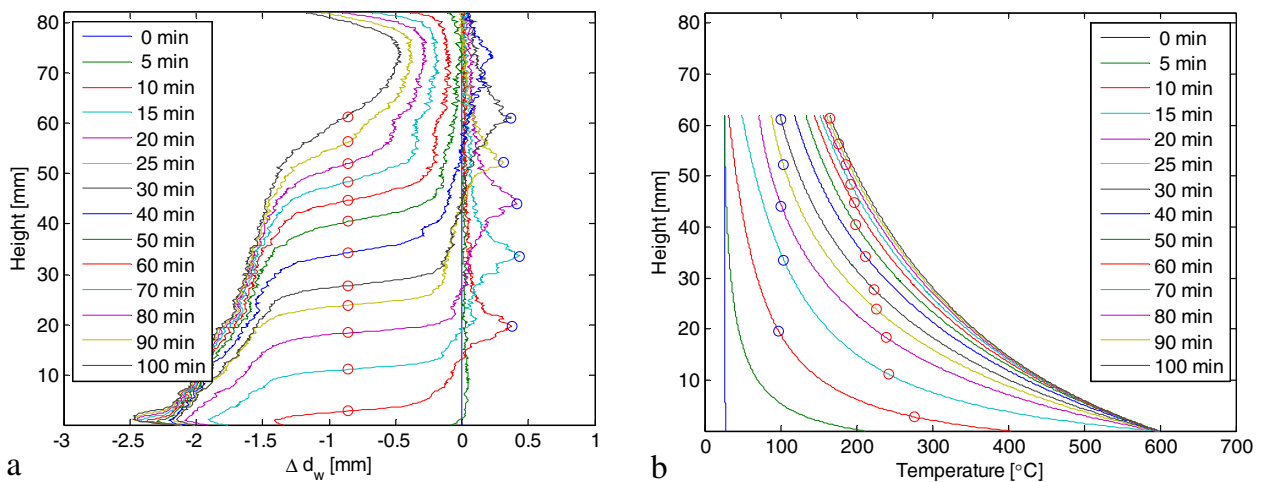

Figure 6. Profiles of a) water change, indicated as mm of water in the cross-section, b) temperature. For the meaning of the red and blue circles, see the text.

Table 1. Compostion of Mortar in mass and volume.

\begin{tabular}{|l|c|c|}
\hline & Mass [g] & Volume [ml] \\
\hline Cement & 100 & 32 \\
\hline Water & 30 & 30 \\
\hline Sand & 109 & 41 \\
\hline Mortar & 239 & 103 \\
\hline
\end{tabular}

Once the free water is removed, dehydration of the mortar is initiated. This endothermic process consumes additional energy, making the moisture profile quite flat. The plateau is indicated by red circles in Figure 6a. The corresponding points are also indicated in the temperature profiles in Figure 6b. The temperature varies from around $250^{\circ} \mathrm{C}$ at the beginning of the experiment to around $160^{\circ} \mathrm{C}$ towards the end of thes test. The first point at $280^{\circ} \mathrm{C}$ seems to be too high, probably because of inaccurate ingterpolation. The temperature range of $160^{\circ} \mathrm{C}-250^{\circ} \mathrm{C}$ is the typical temperature, where most of the dehydration of cement phases occur (CSH, ettringite, and Afm) [14].

Once all the bound water is released, the normal drying process continues. The vapor released during dehydration migrates up to colder regions and condenses there. This manifests itself in the profiles by a peak with positive values (indicated by blue circles in Figure 6a). In the temperature profile, these points are all near $100^{\circ} \mathrm{C}$. Below these points, the temperature is larger than $100^{\circ} \mathrm{C}$ and we can observe a kind of drying front due to boiling of the free water.

Qualitatively, the profiles found by NMR [1] are similar to ours. They also show a sharp drying front and re-condensation behind this front. However, the authors interpret the drying front as boiling of free water. Since the front has a temperature of around $160-195^{\circ} \mathrm{C}$, the corresponding pressure is $0.7-$ 1.4 MPa (not considering capillary pressure). Despite the fact that the heated face reached temperatures up to $350^{\circ} \mathrm{C}$, they observed almost no loss of chemically bound water. The reason might be that the NMR signal shows an irregular behavior for the chemically bound water in the temperature range of $130-250{ }^{\circ} \mathrm{C}[1]$.

For our experiment we exclude the interpretation of the drying front as a result of boiling of evaporable water. The vapor saturation pressure at $200^{\circ} \mathrm{C}$ is around $1.5 \mathrm{MPa}(15 \mathrm{~atm})$, which is unlikely to occur in a specimen of only $10 \mathrm{~mm}$ thickness. However, we can observe a plateau due to boiling of the water accumulated due to condensation. 


\section{CONCLUSIONS}

This preliminary study shows that neutron radiography, due to its high sensitivity to water and sufficient spatial and temporal resolution is a suitable technique to study moisture transport and dehydration of cementitious material subjected to fire.

The main result of the experiment is the evolution of the water loss profile in time. The profile shows a pronounced drying front due to dehydration of cement. This reaction takes place between $180{ }^{\circ} \mathrm{C}$ and $250^{\circ} \mathrm{C}$. An interpretation of the drying front as a consequence of boiling of the evaporable water can probably be excluded, since the corresponding vapor saturation pressure is unlikely to occur in our thin sample.

Above the dehydration front, the profile shows an increase in water content. This increase appears to be due to vapor moving to a colder region and condensing there. At the height of the condensation front, the temperature is around $100^{\circ} \mathrm{C}$.

The authors would like to thank Karim Ghazi Wakili and Marjan Sedighi-Gilani from Empa for making available their test setup and part of their beam time.

\section{References}

[1] van der Heijden G.H.A., Pel L., Adan O.C.G., Fire spalling of concrete, as studied by NMR. Cement and Concrete Research, 42(2), 265-271, 2012.

[2] Justnes H., Bryhn K., Rosvold G.O., Neutron radiography: an excellent method of measuring water penetration and moisture distribution in cementitious materials, Advances in Cement Research, 6(22), 67-72, 1994.

[3] Pleinert H., Sadouki H., Wittmann F.H., Determination of moisture distributions in porous building materials by neutron transmission analysis, Materials and Structures, 31(4), 218-224, 1998.

[4] Hanžič L., Ilić R., Relationship between liquid sorptivity and capillarity in concrete, Cement and Concrete Research, 33(9), 1385-1388, 2003.

[5] de Beer F.C., Strydom W.J., Griesel E.J., The drying process of concrete: a neutron radiography study, Applied Radiation and Isotopes, 61(4), 617-623, 2004.

[6] Kanematsu M., Maruyama I., Noguchi T., Iikura H., Tsuchiya N., Quantification of water penetration into concrete through cracks by neutron radiography Nuclear Instruments and Methods in Physics Research A, 605(1-2), 154-158, 2009.

[7] Zhang P., Wittmann F.H., Zhao T., Lehmann E.H., Neutron imaging of water penetration into cracked steel reinforced concrete Physica B: Condensed Matter, 405(7), 1866-1871, 2010.

[8] Trtik P., Münch B., Weiss W.J., Kaestner A., Jerjen I., Josic L., Lehmann E., Lura P., Release of internal curing water from lightweight aggregates in cement paste investigated by neutron and Xray tomography, Nuclear Instruments and Methods in Physics Research A, 651, 244-249, 2011.

[9] Kanematsu M., Emura G., Tamura M., Tuchiya N., Saito Y., Kawabata Y., Experimental Research on the hydraulic behavior of high strength concrete under high temperature. KURRI progress report 2011, Kyoto University, 2012.

[10] Lehmann E.H., Vontobel P., Wiezel L., Properties of the radiography facility NEUTRA at SINQ and its potential for use as European reference facility, Nondestruct. Test. Evaluat., 16, 191-202, (2001).

[11] Sedighi-Gilani M., Ghazi Wakili K., Koebel M., Hugi E., Carl S., Lehmann E., Visualizing moisture release and migration in gypsum plaster board during and beyond dehydration by neutron radiography, International Journal of Heat and Mass Transfer, 60, 284-290, 2013. 


\section{MATEC Web of Conferences}

[12] Hassanein R., Correction methods for the quantitative evaluation of thermal neutron tomography. Doctoral dissertation, ETH Zurich, Switzerland, 2006.

[13] Hassanein R., Lehmann E., Vontobel P., Methods of scattering corrections for quantitative neutron radiography, Nuclear Instruments and Methods in Physics Research A, 542 (1-3) 353-360, 2005.

[14] Dilnesa B.Z., Lothenbach B., Le Saout G., Renaudin G., Mesbah A., Filinchuk Y. et al., Iron in carbonate containing AFm phases. Cement and Concrete Research, 41(3) 311-323, 2011. 